\title{
Modern Archeological Mapping: Towards Immersive VR Use in Archeology
}

\author{
Alisa Pettitt ${ }^{\mathrm{a}}$, Sven Fuhrmann ${ }^{\mathrm{b}}$

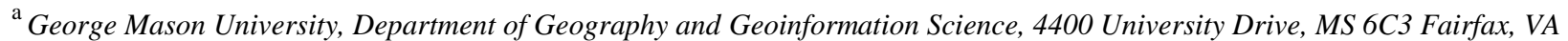 \\ 22030,apettitt@gmu.edu* \\ ${ }^{b}$ George Mason University, sfuhrman@gmu.edu \\ * Corresponding author
}

\begin{abstract}
Detailed mapping is essential to systematic archeological practice and is conducted in five stages: identification, evaluation and excavation, site preservation and documentation, analysis and interpretation, and education. As archeological mapping evolves the five stages remain the same, enhanced by the integration of new geoinformation technologies to better record archeological information. Even with technological advances essential spatial data and mapping products are often overlooked when archeological information reaches the education stage. This disconnect occurs when artefacts are exhibited without considering communicating spatial context, i.e. presented behind glass cabinets. This is a critical concern as understanding spatial context is essential to grasping heritage site histories. As a solution to this wide-reaching issue in geovisualization and the digital humanities, this research proposes a framework for developing immersive virtual reality heritage site applications that preserve the spatial context of archeological mapping. Applying the framework, this research produced the heritage site application "VR Riverbend", which successfully relinks archeological materials with essential spatial context. Thus, VR Riverbend connects archeological data with place, remedying issues of artefact/context disconnect inherent in traditional exhibits. Developing immersive VR heritage site applications following the introduced framework provides a solution towards effective spatial and often multi-layered archeological heritage site education.
\end{abstract}

Keywords: Archeological Mapping, Digital Humanities and GIS, Archeology, Virtual Reality, Geovisualization

\section{Introduction}

Mapping, at different scales, is completed during all stages of archeological site discovery, assessment and interpretation. The importance of maps and related geoinformation technologies for archeological field studies is unquestionable. Archeological field practice demands a systematic approach involving a thorough comparison of historic recordings, i.e. narratives, sketches, imagery, etc. with the existing physical landscape. Banning (2002) breaks archeological practice into five significant categories: site location, site assessment and site excavation, site preservation and documentation, site interpretation, and site curation and education. These categories require several surveying, scanning and mapping techniques, thus providing new requirements and opportunities in archeology and cartography for crossdisciplinary research.

Best practices in archeological mapping have evolved from hand-mapping broad site boundaries to pinpointing archeological data with Global Navigation Satellite Systems (GNSS) receivers. New tools such as unmanned aerial vehicles (UAVs), accessible photogrammetry software, and 3D geoinformation technologies combined with traditional cartographic archeological processes are resulting in superior visualizations for conveying archeological information. In particular, the current movement towards collecting and creating threedimensional data is altering how archeologists conceive of and present archeological maps. 3D archeological site data provide highly accurate, unbiased heritage site information that can serve a myriad of purposes including but not limited to creating engaging geovisualization displays.

With these content-rich representations archeologists possess new opportunities for analyzing and visualizing important archeological information. Nowadays, the problem is often not lack of data but managing and interpreting data. As in other disciplines, the questions of how to process and convey this berth of information need to be addressed. Advances in immersive virtual reality (VR) technologies are one approach towards visualizing high-dimensional archeological data for site discovery, assessment, interpretation and education. Mobile VR systems, e.g. the Oculus Go or the Lenovo Mirage Solo are in the consumer market and custom development environments for VR applications have become accessible. These mobile, immersive systems provide opportunities to discover, assess and interpret multilayered archeological data, in particular, 3D data sets. 
Infusing traditional archeological mapping with new spatial data types in immersive, mobile VR environments is a powerful means of conveying and educating on archeological resources. VR platforms that allow users, experts and the general public alike, to explore multiple components and data layers of heritage sites offer opportunities to explore archeological information, heritage and history on higher levels. Digital preservation, interpretation, analysis, and education on and at archeological sites can be greatly enhanced through VR technologies. In particular in the education stage of the archeological process VR technologies can be harnessed to remedy the disconnect between spatial context and archeological materials, often found in traditional museum exhibits.

Merging traditional archeological mapping with VR is an important future iteration of archeological practice. This manuscript presents a framework for developing VRbased heritage site education, applied at Riverbend Park in Northern Virginia. Riverbend Park contains significant archeological sites, data from these sites presented in programs used for educating Virginia's school children about physical geography and lifeways of Native Americans, past and present (VDOE 2008). This research uses Riverbend Park as a test case to document and assess various heritage site datasets, discuss archeological data collection and object modelling methods, and describe the workflow for developing educational VR applications. The findings and design suggestions of this project can be utilized and transferred to other national and international heritage sites for history education and for creating exhibits that better communicate context and the multidimensional nature of archeological resources.

\section{Archeological Mapping with Geoinformation Technologies}

Between archeology and cartography there has always existed a complementary relationship. Understanding human-environment interactions in ever-evolving landscapes is essential for interpreting and understanding cultures, heritage and history. Mapping in archeological practice begins before conducting field work through intensive background research, continues during site excavation and interpretation and concludes in visualizing interpreted findings to archeologists, historians, anthropologists, the general public and other domains. Mapping and the interpretation of archeological data are crucial to modern archeological practice and findings from investigations without accurate spatial context lose layers of meaning.

\subsection{Identification}

The initial step of archeological mapping is site identification. Urban and transportation developments often serve as driving forces behind archeological investigations, proceeding construction processes to ascertain presence/absence of archeological resources. In advance of field work, a comprehensive review of primary and secondary sources concerning the site including maps, aerial photography, and historical documents is completed.

If archeological materials are present on the landscape surface, preliminary steps of site identification can be conducted without breaking ground. In Virginia, this involves registering the site with the state at which point the site receives a unique identifier. If no archeological surface materials have been identified, often a grid is constructed using a cartesian coordinate grid-system which is placed over the property. This grid is comprised of unique points which represent small test holes to be excavated, spaced at an interval and size following standards established by the state. If a property is assessed and it is found that archeological excavations are necessary, excavations will commence by first completing tests contained within the cartesian grid. The test sites are marked in the field using surveying tools ranging from traditional transits to theodolites and high-end receivers. Meticulous mapping of these points in a GIS is crucial to understanding context of discovered archeological materials and also in locating identified areas of interest during future work.

\subsection{Evaluation and Excavation}

Excavating test sites within the cartesian grid is the first step in archeological field practice for investigating an unknown site. During the initial mapping process, focus is on recording the horizontal extent of the site. When ground-disturbing excavations commence, site mapping begins to document the vertical extent. Recording vertical data typically involves documenting the stratigraphic sequence encountered in tests, traditionally recorded through hand-mapping. Nowadays, photogrammetry software can provide 3D data of stratigraphic sequences at high accuracy. The stratigraphic sequence is essential for understanding landscape evolution as well as artefacts and features identified in individual strata. When the recovered artefacts are processed in the laboratory, mapping their provenience showing vertical and horizontal context is essential for planning further work. At this stage, maps detailing material context are used to identify artefact hot spots and determine if spatial patterns are present.

\subsection{Site Preservation and Documentation}

Archeological mapping methods have evolved from conventional hand-mapping techniques and traditional photography to the incorporation of tools such as photogrammetry (Lambers et al. 2007; Lerma et al. 2010; Fernández-Hernández et al. 2015) and 3D scanning (Karasik and Smilansky 2008; Forte et al. 2012). Remote sensing data that has greatly aided archeological discovery and mapping processes include Lidar, Radar, Radarsat, newer high-resolution satellite data (e.g. Quickbird), and legacy data from platforms such as Landsat (Parcek 2009; Luo et al. 2014; Agapiou and Lysandrou 2015). More and more unmanned aerial vehicles (UAVs) outfitted with a variety of sensor equipment are employed at archeological sites to rapidly map them (Remondino et al. 2010). Especially for remote and inaccessible areas, UAVs are 
emerging as valuable assets for mapping previously unreachable archeological sites.

For heritage site preservation and documentation, these technologies are essential to provide an accurate account for site significance assessment and later dissemination to national and international experts. In particular, current developments in 3D GIS greatly enhance the documentation and interpretation of archeological sites. Platforms that offer 3D mapping, such as ArcGIS Pro or QGIS, alter the way archeologists think about site preservation and allow experts to share and collaborate online.

If a heritage site cannot be protected from natural elements, human interaction, or is in danger of deterioration, procedures for site mitigation are developed. Sometimes such work involves full-scale data recovery excavations in which as much archeological data is collected as possible. Accurate site recording is essential and modern archeological mapping employs digital technologies, i.e. transit levels, laser theodolites, various image-based modeling (IBM) techniques, or 360-degree videos to digitally document sites. Digital site preservation is crucial in modern archeological mapping. Developing digital technologies that meticulously document and preserve site data is a major issue in the cultural heritage domain (Fresa 2013; Wells et al. 2014; Povroznik 2018). This priority is also reflected across national and international culture heritage groups and builds an important future iteration of archeological mapping (Historic England 2017; Department of Arts, Heritage, Regional, Rural and Gaeltacht Affairs 2017).

\subsection{Analysis and Interpretation}

Effective heritage site analysis and interpretation is achieved by processing accurately collected data. This stage is greatly aided by geoinformation technologies, which map and store site locations and attribute information in geospatial databases. GIS are utilized to combine different data types and media such as handdrawn maps, stratigraphic and artefact data. These content rich datasets provide additional heritage information that enhance GIS-based analysis and interpretation. The Commonwealth of Virginia maintains the Virginia Cultural Resource Information System (VCRIS), a GIS containing all recorded archeological sites in Virginia. Other U.S. States and countries have similar archives established, often allowing cross-references about investigation methods, identified artefacts, and other content.

Besides utilizing GIS for interpretation, archeological mapping is moving towards collecting/creating custom 3D visualizations with computer animation and modelling software, i.e. Autodesk's Maya and Blender. 3D archeological mapping across different scales, from objects to landscapes, is becoming a major component of the archeological interpretation process. This process is enhanced by a joint effort of archeologists, cartographers, historians and 3D artists that aim to visualize how archeological features may have looked in the past by ensuring that these models serve as knowledge representations agreed on by more than one expert or artist (Rua and Alvito 2011). These virtual representations serve as models for scientific discussion and can be altered to represent the collective scientific community's findings. Emergent technologies also allow for the incorporation of alternative site interpretations and narratives to encompass multiple perspectives of data in these $3 \mathrm{D}$ archeological site mappings. As archeological mapping advances, 3D computer animation and modelling become more important and serve as a technology to better understand content-rich or even fragmented archeological data.

\subsection{Education}

Educating about heritage sites is the final stage in archeological practice. There are many methods of conveying knowledge recovered from sites to the public; one is through museums and exhibitions. Museum educators serve as stewards for presenting and explaining archeological information. Exhibitions focused on showcasing archeological objects and sites aim to connect with the general public through highlighting special artefacts, cultural lifeways and rituals through novel and engaging approaches. Museum educators act as gatekeepers as they decide which artefacts to present and which histories from possibly several narratives to communicate. A major challenge in creating exhibits is identifying which information to show and which media to use. In traditional museums exhibits information is often presented where a disconnect exists between archeological materials and associated provenience.

This disconnect often stems from early museum exhibition practice, where exhibits served as "cabinets of curiosities." These displays highlighted interesting materials collected from expeditions, with exhibits often lacking spatial and cultural context. Unfortunately, this legacy has been inherited by modern museums, many institutions still presenting exhibits to the public where artefacts are separated from provenience. When historic artefacts are separated from their spatial context the exhibits lose their deeper historic and cultural meaning. This observation is criticalbecause up until the education stage, archeological mapping always captured, analyzed and interpreted artefacts and landscapes tied to spatial data. Negating to present the spatial context in heritage displays demotes the importance of this systematic science and does not communicate a complete picture of the archeological site, the artefacts and associated narratives.

VR technologies are one possible solution in reconnecting and showing the spatial context linked to archeological artefacts in museum exhibits. At its core, VR can enhance a visitor's appreciation of archeological materials by assisting in visualizing the artefact in a scene and informing the audience on the importance of archeological mapping in field practice. Spatial and cultural context are essential components to archeological practice and conveying this notion to the public could aid in better grasping heritage and history. Artefacts 
spotlighted behind glass cases are beautiful to look at, but this presentation style disregards context which is fundamental in fully understanding the remains of past peoples.

The five stages of archeological mapping detailed above outline the importance of accurately collecting and documenting spatial information throughout the entire archeological process. As a result, a wealth of historic data is collected that allows for analysis and interpretation by experts and outreach and education for the general public. Especially for public education VR technology has started to show major impacts on how new museum exhibitions are curated. Recent successful examples highlight how spatial data connected to archeological materials can be effectively communicated through VR displays (Barbieri et al. 2017; Pollalis et al. 2018; Schofield et al. 2018).

Interactive, immersive VR platforms can assist in connecting broader audiences with narratives on heritage sites and serve as successful vehicles for history education. These technological advances have reached the consumer market and are more accessible to heritage sites to introduce effective, innovative geovisualization displays that provide novel, engaging narratives to the public. The following section proposes a framework for developing VR-based heritage site education applications. As an example, the framework is applied to "VR Riverbend", a VR exhibit created for a cultural resource park in Northern Virginia. VR Riverbend demonstrates how spatial context reconnected with associated archeological materials convey more holistic presentations for museum exhibits.

\section{VR Framework for Heritage Sites: Education- focused Case Study for Riverbend Park}

Riverbend Park, a resource-based park located in Virginia's Fairfax County, is situated on the Potomac River, approximately twenty miles northwest of Washington D.C. Riverbend Park contains nearly 100 archeological sites with history spanning over 10,000 years. Within the park, select artefacts from Riverbend are exhibited so visitors can connect with Riverbend's history and learn about early lifeways in Virginia. In addition, the museum exhibits art created by descendants of indigenous peoples of this region. Riverbend Park is vested in conveying the importance of Virginia's Native American history and in addition to permanent exhibits hosts several educational programs on regional Native American history, e.g. an annual Indian Festival every fall.

Visualizing heritage site histories, such as those tied to Riverbend, can be challenging. This is especially true when tangible histories are hidden below the surface. Archeological data associated with heritage sites is typically meticulously mapped but often archeological finds are housed in displays that disregard scrupulously mapped context. For some sites, pinpointing artefact provenience could be hazardous, as looting at heritage sites is a significant issue. However, for sites where full data recovery has occurred and finds are safely stored in collections, connecting context with displayed archeological data can serve to enhance understanding of the materials and heritage site. Most heritage sites possess rich geospatial data sets to create displays illustrating the multifaceted meanings tied to artefacts. These data layers can be presented in conjunction with archeological materials by embracing VR technologies to craft exhibits that better communicate context.

A goal of this research was to develop a replicable framework for immersive VR applications for better conveying multidimensional layers embedded in archeological data. Many heritage sites already have these data types collected from traditional and modern archeological mapping techniques discussed above. The proposed framework consists of four stages, applying different techniques and technologies: Narrative Development, 3D Game Engine Development, Advanced Media, 3D Modelling and Animation, and Head Mounted Display VR Implementation (Figure 1). Following this framework, the research produced VR Riverbend, an immersive VR display presenting archeological materials with contextual data to aid users in developing deeper appreciation and understanding of archeological data and heritage sites.

\begin{tabular}{|c|c|c|}
\hline \multicolumn{3}{|c|}{ Narrative Development } \\
\hline \multicolumn{3}{|c|}{$\begin{array}{c}\text { Archaeological reports, Historical documents, Input from } \\
\text { experts and descendants of indigenous cultures }\end{array}$} \\
\hline \multicolumn{3}{|c|}{ Game Engine Development } \\
\hline \multicolumn{3}{|c|}{ Advanced Media, 3D Modelling, and Animation } \\
\hline $\begin{array}{c}\text { Static 3D Objects } \\
\text { Artifacts and } \\
\text { Archaeological Features }\end{array}$ & $\begin{array}{l}\text { Animate 3D Objects } \\
\text { Humans, Animals } \\
\text { Animated Artifacts }\end{array}$ & $\begin{array}{l}\text { Environment } \\
\text { Terrain, Water, } \\
\text { Atmosphere }\end{array}$ \\
\hline$\checkmark$ & $\checkmark$ & $V$ \\
\hline $\begin{array}{l}\text { Photogrammetry, } \\
\text { 3D scanning, } \\
\text { 3D creation suites }\end{array}$ & $\begin{array}{l}\text { 3D Humanoid Modeling } \\
\text { Software, } \\
\text { Animation Software }\end{array}$ & $\begin{array}{l}\text { Laser Theodolites, } \\
\text { GPS Recievers, Lidar, } \\
\text { Radar, Procedural Worlds } \\
\text { Assets }\end{array}$ \\
\hline \multicolumn{3}{|c|}{ Virtual Reality Implementation } \\
\hline $\begin{array}{l}\text { Desktop } \\
\text { Virtual } \\
\text { Reality }\end{array}$ & $\begin{array}{c}\text { Head } \\
\text { Mounted } \\
\text { Devices (HMD) }\end{array}$ & $\begin{array}{c}\text { Cave Automated } \\
\text { Virtual } \\
\text { Environments (CAVE) }\end{array}$ \\
\hline
\end{tabular}

Figure 1. VR Framework for Heritage Sites

\subsection{Narrative Development}

The first stage in this framework is writing a detailed narrative which outlines content and context of the exhibit. This important step is typical of modern exhibit design, including VR displays. To craft the VR Riverbend narrative several steps were followed, most that can be readily adopted by other heritage sites. Close collaboration with an advisory team comprised of Fairfax County archeologists, employees of Riverbend, instructors from local schools, and descendants of regional indigenous peoples, produced an extensive historic background which served as a guide for later steps in exhibit development.

During this stage, the assembled team identified appropriate materials for developing this narrative which included: background histories, archeological notes and reports, optimal primary and secondary sources, and 
spatial data types from archeological mappings ranging from hand-drawn maps to data collected from high-end GPS receivers. Thomas de Bry and John White imagery from the sixteenth century was particularly useful for creating characters and scenes for this VR environment (Figure 2). These images were evaluated with other primary sources and the advisory team in efforts to construct a narrative inclusive of multiple perspectives.

Creating a solid narrative is an essential first step in this development framework as it sets the theme of the VR display and identifies histories and archeological data best suited for VR display.

\subsection{D Game Engine Development}

The second stage in this framework focuses on 3D Game Engine Development. Several game engines are available to develop immersive VR experiences and games engines have become a common application in modern VR geovisualization. Over the past several years Unity has emerged as the leading cross-platform game engine for VR application development. This platform can be utilized to develop 2D and 3D gaming applications and has been successfully used to visualize almost any type of spatial data. Several examples for Unity applications in the geovisualization context exist, e.g. Schofield et al. (2018) and Bruno et al. (2018). A platform assessment resulted in selecting Unity $3 \mathrm{D}$ as development platform for VR Riverbend: a flexible, customizable development environment, multi-platform development options, 3D assets, and a large, active online community.

As 3D object and environment modelling is complex and time consuming, the Unity asset database offers access to detailed 3D models, such as realistic vegetation, atmospheres, characters, etc. This model database was a valuable resource in developing the VR Riverbend environment. In several instances well-crafted 3D models were available through the asset database matching the needs of the VR application. Additional functional enhancements, such as audio resources helped enrich user experience with immersive soundscapes such as a rushing Potomac river, crackling fire pits, and sounds of fauna. The Unity developing environment was a good solution for remedying the issue of spatial disconnect with archeological data, providing a solid cross-platform for linking narratives, scanned and modelled artefacts, and landscape features. This development platform can be readily accessed by heritage sites with interest in constructing custom displays using VR technologies and Unity's cross-development platform properties support the majority of available VR and gaming platforms. These broad development options combined with an accessible game engine offer new opportunities for heritage sites to expand on VR displays to future iterations including augmented reality (AR) and mixed reality (MR) applications.

\subsection{Advanced Media, 3D Modelling and Animation}

For archeological mapping purposes, modelling in the Unity environment requires utilization of a range of data types and media. Three major components were identified for educational heritage site VR development: 360-degree video, photogrammetry, and 3D modelling and life-like animation. This stage was overseen by the advisory team, the developed narrative outlining artefacts, art and landscape elements necessary for producing a realistic and accurate VR environment. Counsel from the advisory team combined with 360-degree video, photogrammetry, 3D digital models animated characters, and rich spatial data were blended in Unity to create a rich VR experience.

\subsubsection{0-Degree Video}

360-degree video is an effective technique of quickly integrating informative, interactive video scenes into VR displays. Collecting detailed imagery is crucial to archeological documentation and cameras are essential components in the archaeologist's toolkit. In addition to

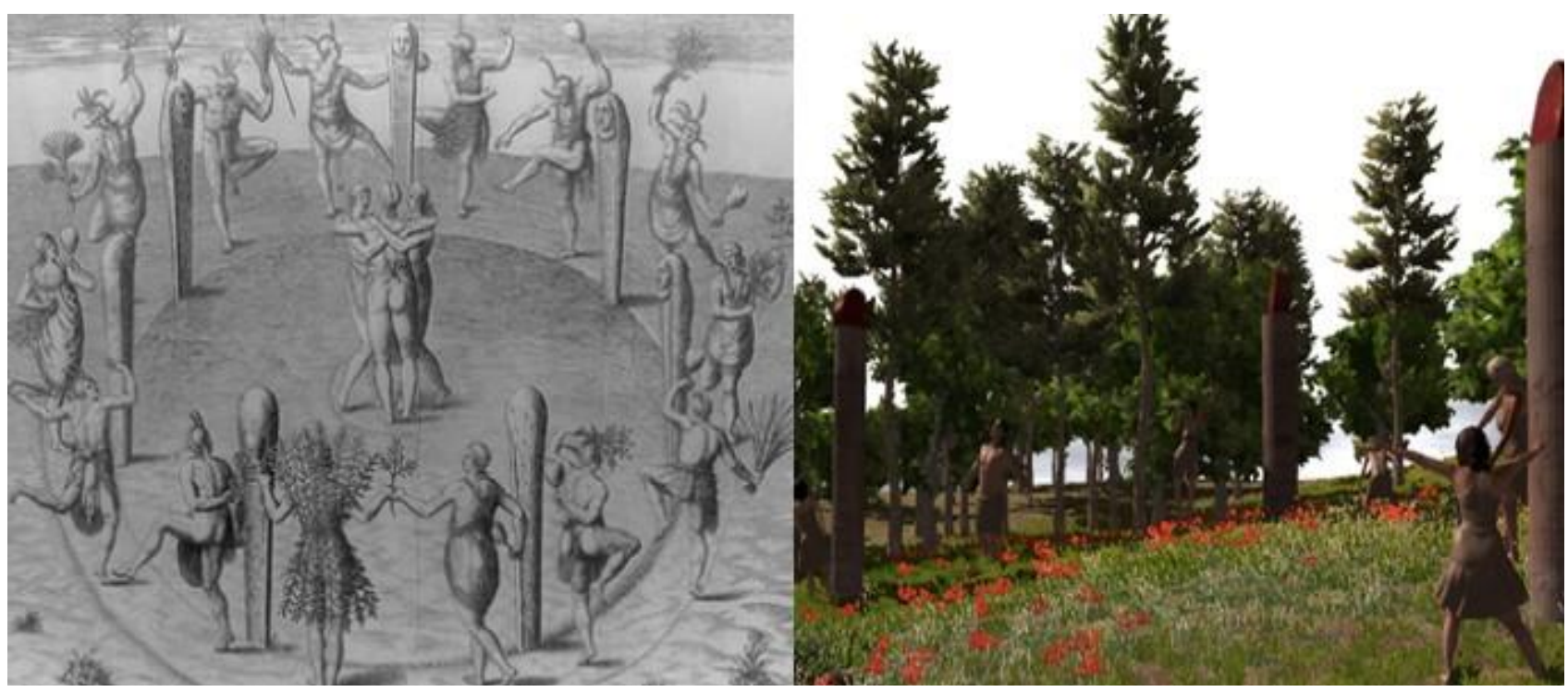

Figure 2. "The dances at their great feast" (Bry and White 1590) with corresponding narrative in VR Riverbend 
traditional photography archeologists are embracing 360degree video for quick, unbiased capture of sites (Dempsey 2015). These scenes can be seamlessly integrated into custom, interactive VR exhibits for heritage site education.

In VR Riverbend, 360-degree video was used to inform audiences about the site's history and also aid users in better understanding physical features in the actual park. An example, when users enter the virtual wigwam in VR Riverbend they experience a 360-degree video of the inside of Riverbend's replica wigwam. They can experience the wigwam in a furnished state in VR Riverbend which they typically would not encounter during a park visit. 360-video is an accessible, userfriendly technology that can be easily harnessed by heritage sites for creating quick, educational materials for display in VR.

\subsubsection{Photogrammetry for Virtual Artefact Creation}

Advances in photogrammetry software allow for the rapid creation of accurate 3D models of archeological data types including artefacts and cultural landscapes. Integrating detailed 3D artefact models allows users to see and interact with fragile materials, normally inaccessible in collections or in museum exhibits. This modelling process also allows for the display of smaller, often overlooked but important materials such as pottery sherds. Precise 3D models of pieces of artefacts can also be displayed in VR as they may have looked in complete form e.g. a pottery sherd as part of a whole pot. This approach offers audiences to immediately visualize archeological materials in more complete forms which could assist in overall understanding of the artefact's purpose.

This interaction with usually inaccessible artefacts connects the public to Riverbend's past landscape and educates on unfamiliar artefacts. In contrast to traditional exhibitions, the public can experience artefacts in the context from which they were removed, essential in developing deeper understandings of artefacts and archeological sites. Immersive and interactive VR exhibits can brush the dust of artefacts in closed collections and allow the public to experience the details and uniqueness of each piece. Reconnecting artefacts with their spatial context is important for showing the multiple data layers associated with these materials. Highlighting this important connection between archeological data and spatial context could connect application users to cultural landscapes and foster a greater appreciation of heritage sites.

\subsubsection{D Modelling and Life-Like Animations}

360-degree video and accurate 3D models of archeological materials are components many archeologists already collect in modern mapping methods (Forte et al. 2012; Fernández-Hernández et al. 2015; Dempsey 2018). A necessary component that cannot be collected from mapping processes is the interpretive data necessary for visualizing past environments. These data types cannot be captured in the present with cameras and sensors and require 3D artistry skill for developing realistic handcrafted models. At this stage, counsel from advisory team is essential as these $3 \mathrm{D}$ data types represent an interpretive version of the heritage site's past environment.

Creating hand-crafted models can be a daunting and timeconsuming process. Recently, programs to assist developers in creating custom models have emerged which accelerate this task. Adobe Fuse, a creation software that aids in building life-like human models was used in developing VR Riverbend. Human models created in Fuse were uploaded into Mixamo, a software that rapidly rigs and animates characters. Fuse and Mixamo helped produce realistic characters ready for integration into the application (Figure 3). Adding animated characters to virtual environments enhances VR visualizations with vivid and sometimes interactive models. These models can be easily altered to fit unique needs of heritage sites. Technological advances in 3D modeling are trending towards creating solutions for expediting the modeling process, such as Fuse and Mixamo. Another quickmodeling program, Gaia, rapidly produced a realistic procedural world creating a lush, forest environment for VR Riverbend.

To add custom clothing, jewelry, tattoos, and hairstyles to VR Riverbend models were edited in Blender, an opensource 3D creation suite. Blender was also used to create custom models for this application, such as dugout canoes. Blender is one of many 3D creation suites institutions could utilize in application development but is one of the only open-source options, a great incentive for often resource-impaired heritage sites.

Blender, Fuse, Mixamo, and Gaia assisted in developing VR Riverbend's custom 3D models. For custom character animations, this project developed animations using Blender, Xbox Kinect, and Ni-Mate. Xbox Kinect devices are motion-sensing systems that can be combined with $\mathrm{Ni}$ Mate, a motion tracking program, in Blender 3D to create custom animations. These programs were used to develop basic animations of Native American dances, which will be extended with the help and support of regional tribes. Integrating dances captured by descendants of Virginia's indigenous peoples will assist in digitally preserving intangible elements associated with past cultures.

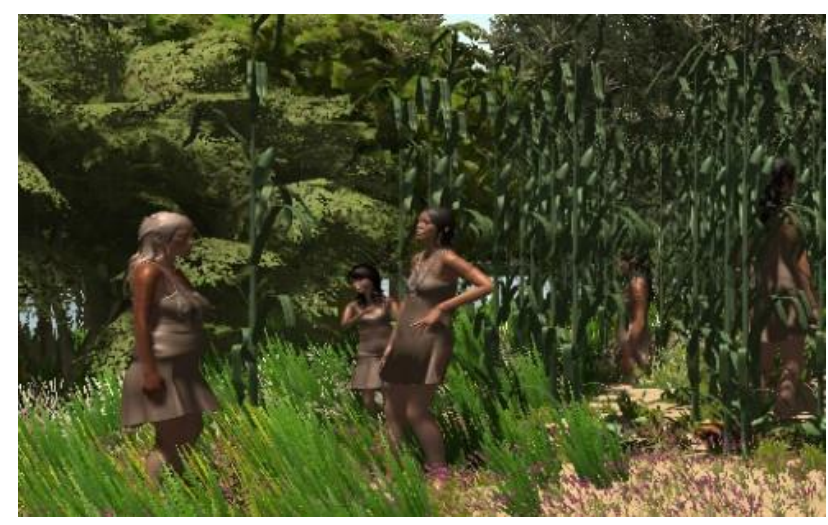

Figure 3. Characters from VR Riverbend 
Identifying appropriate materials to display and gathering appropriate permissions to display unique dances is planned for future iterations of VR Riverbend.

Advanced media, 3D modelling, and animations can breathe life into archeological data through VR displays. Many of these data types are already collected in modern archeological mapping and can be readily merged into immersive VR environments. VR displays can show tangible and intangible components of cultural heritage and reveal some of the different facets tied to archeological data. In particular, VR applications can connect data types with associated spatial context, alleviating issues with disconnect and assisting users in better understanding sites and space. As archeological mapping continues to embrace new technologies data types suitable for VR display will become available for integration into custom VR visualizations used to highlight the multidimensional nature and significance of heritage sites.

\subsection{Head Mounted Display VR Implementation}

For displaying immersive VR, head mounted displays (HMD), such as Oculus's Go or HTC's VIVE, have reached the consumer market. These immersive platforms provide rich 360-degree experiences for users and are selfcontained systems or run off gaming laptops, a portability characteristic permitting easy transport to museums and other educational settings. Purchasing immersive systems such as the Oculus Rift is not essential for sites and museums wanting to display VR applications, heritage sites can also display VR exhibits across a variety of media platforms from desktop computers to mobile devices. However, the immersive component offered in HMD's is still novel for many users and could entice new visitors to sites. Also, immersive VR displays immediately connect users to the place which could lead to a greater comprehension of the importance of spatial data at sites. VR users can virtually stand in past landscapes and interact with typically inaccessible archeological materials. In HMD-based VR, artefacts are presented outside their glass display cases and placed in context. Immersive VR displays provide settings for artefacts to seize meanings stripped from them in traditional exhibits. As HMDs reach attainable prices heritage sites can acquire these technologies for creating custom VR displays that immerse visitors in the past and connect audiences with rich archeological data layers. VR Riverbend was created following the framework outlined above, demonstrating that different archeological data types can be melded into educational exhibits that communicate context and engage users with rich multidimensional layers intrinsically linked to heritage sites.

\section{Conclusions}

This research proposes immersive VR exhibits as one solution for maintaining crucial connections between archeological materials and associated spatial context often overlooked in the education stage of archeological mapping. 3D data sets collected during modern archeological mapping provide ample source material for developing immersive, interactive VR heritage site experiences, presented in this research through VR Riverbend. VR Riverbend is currently in preliminary phases of user-testing, garnering feedback from leaders in Virginia's archeological community (state archeologists, academics, regional tribes) to assess if goals to communicate connections between archeological materials and spatial context were achieved. The initial testing has been conducted at meetings such as the Middle Atlantic Archeology and Archeological Society of Virginia conferences and Riverbend Park's annual Indian Festival.

A main objective of this research was to create a development framework for VR-based education for heritage sites that can be applied to other national and international sites. The framework consists of four stages, applying different techniques and technologies: Narrative Development, 3D Game Engine Development, Advanced Media, 3D Modelling and Animation, and Head Mounted Display VR Implementation. This framework and applied methods and technologies demonstrate that the areas of cartographic and archeological mapping provide a solid foundation to further explore this topic. In both domains, technological advancements and increasingly accessible development platforms provide scientists, not only in the in the cultural heritage sector, with educational opportunities that could attract new audiences and promote heritage site conservation.

Emergent VR technologies offer opportunities to present new narratives stressing the importance of context and illustrating the essential relationship between mapping and systematic archeological practice. VR Riverbend, developed following the framework presented in this research, demonstrates VR as an effective platform for displaying this complementary relationship and can be aptly applied to other heritage sites. This research illustrated that VR technologies can be harnessed by heritage sites for communicating context and better conveying the wealth and breadth of knowledge embedded in archeological data.

\section{References}

Agapiou, A., \& Lysandrou, V. (2015). Remote sensing archeology: Tracking and mapping evolution in European scientific literature from 1999 to 2015. Journal of Archeological Science: Reports, 4, 192-200.

Barbieri, L., Bruno, F., \& Muzzupappa, M. (2018). Usercentered design of a virtual reality exhibit for archeological museums. International Journal on Interactive Design and Manufacturing (IJIDeM), 12(2), 561-571.

Bruno, F., Barbieri, L., Lagudi, A., Cozza, M., Cozza, A., Peluso, R., \& Muzzupappa, M. (2018). Virtual dives into the underwater archeological treasures of South Italy. Virtual Reality, 22(2), 91-102.

Bry, T. and White, J. (1590). "The dances at their great feasts." Library of Congress. Library of Congress Rare Book and Special Collections Division Washington, D.C. 
Dempsey, Andrew. (2015). Suspected 1665 Great Plague Pit Unearthed at Crossrail Liverpool Street Site. Crossrail. August 12, 2015.

Department of Arts, Heritage, Regional, Rural and Gaeltacht Affairs. (2017). Culture 2025: Eire Ildanach: A Framework Policy to 2025 Draft Document. Ireland's Department of Arts, Heritage, Regional, Rural and Gaeltacht Affairs.

Fernández-Hernández, J., González-Aguilera, D., Rodríguez-Gonzálvez, P., \& Mancera-Taboada, J. (2015). Image-Based Modelling from Unmanned Aerial Vehicle (UAV) Photogrammetry: An Effective, LowCost Tool for Archeological Applications: Image-based modelling from UAV photogrammetry. Archaeometry, 57(1), 128-145.

Forte, M., Dell’Unto, N., Issavi, J., Onsurez, L., \& Lercari, N. (2012). 3D Archeology at Çatalhöyük. International Journal of Heritage in the Digital Era, 1(3), 351-378.

Fresa, A. (2013). A Data Infrastructure for Digital Cultural Heritage: Characteristics, Requirements and Priority Services. International Journal of Humanities and Arts Computing, 7(supplement), 29-46.

Historic England. (2017). Research Agenda. Swindon: Historic HistoricEngland.org.uk/research/agenda England.

Karasik, A., \& Smilansky, U. (2008). 3D scanning technology as a standard archeological tool for pottery analysis: practice and theory. Journal of Archeological Science, 35(5), 1148-1168.

Lambers, K., Eisenbeiss, H., Sauerbier, M., Kupferschmidt, D., Gaisecker, T., Sotoodeh, S., \& Hanusch, T. (2007). Combining photogrammetry and laser scanning for the recording and modelling of the Late Intermediate Period site of Pinchango Alto, Palpa, Peru. Journal of Archeological Science, 34(10), 17021712.

Lasaponara, R., \& Masini, N. (Eds.). (2012). Satellite remote sensing: a new tool for archeology. Dordrecht ; New York: Springer.

Lerma, J. L., Navarro, S., Cabrelles, M., \& Villaverde, V. (2010). Terrestrial laser scanning and close range photogrammetry for 3D archeological documentation: the Upper Paleolithic Cave of Parpalló as a case study. Journal of Archeological Science, 37(3), 499-507.

Luo, L., Wang, X., Liu, C., Guo, H., \& Du, X. (2014). Integrated RS, GIS and GPS approaches to archeological prospecting in the Hexi Corridor, NW China: a case study of the royal road to ancient Dunhuang. Journal of Archeological Science, 50, 178-190.

National Register of Historic Places (NRHP) (2002). National Register Bulletin. Washington D.C. United States Department of Interior.

Parcak, S. H. (2009). Satellite remote sensing for archeology. London; New York: Routledge.

Povroznik, N. (2018). Towards a Global Infrastructure for Digital Cultural Heritage. In M. Ioannides, E. Fink, R.
Brumana, P. Patias, A. Doulamis, J. Martins, \& M. Wallace (Eds.), Digital Heritage. Progress in Cultural Heritage: Documentation, Preservation, and Protection (Vol. 11196, pp. 607-615). Cham: Springer International Publishing.

Remondino, F., Barazzetti, L., Nex, F., Scaioni, M., \& Sarazzi, D. (2012). UAV photogrammetry for mapping and $3 \mathrm{~d}$ modeling - current status and future perspectives. ISPRS - International Archives of the Photogrammetry, Remote Sensing and Spatial Information Sciences, XXXVIII-1/C22, 25-31.

Rua, H., and P. Alvito. 2011. "Living the Past: 3D Models, Virtual Reality and Game Engines as Tools for Supporting Archeology and the Reconstruction of Cultural Heritage - the Case-Study of the Roman Villa of Casal de Freiria.” Journal of Archeological Science 38 (12): 3296-3308.

Pollalis, C., Minor, E. J., Westendorf, L., Fahnbulleh, W., Virgilio, I., Kun, A. L., \& Shaer, O. (2018). Evaluating Learning with Tangible and Virtual Representations of Archeological Artifacts. In Proceedings of the Twelfth International Conference on Tangible, Embedded, and Embodied Interaction - TEI '18 (pp. 626-637). Stockholm, Sweden: ACM Press.

Schofield, G., Beale, G., Beale, N., Fell, M., Hadley, D., Hook, J., ... Thresh, L. (2018). Viking VR: Designing a Virtual Reality Experience for a Museum. In Proceedings of the 2018 on Designing Interactive Systems Conference 2018 - DIS '18 (pp. 805-815). Hong Kong, China: ACM Press.

Wells, J. J., Kansa, E. C., Kansa, S. W., Yerka, S. J., Anderson, D. G., Bissett, T. G., ... Carl DeMuth, R. (2014). Web-based discovery and integration of archeological historic properties inventory data: The Digital Index of North American Archeology (DINAA). Literary and Linguistic Computing, 29(3), 349-360.

Virginia Department of Historic Resources (VDHR 2017). Virginia Cultural Resource Information System (VCRIS). Commonwealth of Virginia. Richmond, Virginia.

Virginia Department of Education (VDOE) (2018). History and Social Science Standards of Learning. Commonwealth of Virginia. Richmond, Virginia. 